The Journal of Symbolic Logic

Volume 63, Number 2, June 1998

\title{
ON THE COMPUTATIONAL CONTENT OF THE AXIOM OF CHOICE
}

\author{
STEFANO BERARDI, MARC BEZEM, AND THIERRY COQUAND
}

\begin{abstract}
We present a possible computational content of the negative translation of classical analysis with the Axiom of (countable) Choice. Interestingly, this interpretation uses a refinement of the realizability semantics of the absurdity proposition, which is not interpreted as the empty type here. We also show how to compute witnesses from proofs in classical analysis of $\exists$-statements and how to extract algorithms from proofs of $\forall \exists$-statements. Our interpretation seems computationally more direct than the one based on Gödel's Dialectica interpretation.
\end{abstract}

Introduction. It is well-known that the Axiom of Choice [16] is validated by the Brouwer-Heyting-Kolmogorov explanation of the logical constants [3]. In view of the negative translation of classical arithmetic into intuitionistic arithmetic $[13,6]$, one would expect that it is possible to make constructive sense of the Axiom of Choice as used in informal mathematics, for instance in the use of Zorn's Lemma, or in establishing the existence of a well-ordering on the reals.

This, however, appears to be non-trivial. The combination of the Axiom of Choice and the Excluded Middle turns out to be extremely problematic from a constructive point of view. To make constructive sense of such a combination can actually be seen as one of the main aims of Hilbert's programme $[8,9,1]$. We address here the more modest question of the analysis of the computational content of the Axiom of Choice, by giving a novel realizability interpretation of the negative translation of the Axiom of (countable) Choice. This interpretation is due to the third author, motivated by [5].

Most of the work cited above has been inspired by metamathematical questions (consistency proofs, proof theoretic strength). Quite a different motivation arises from the computer science point of view, namely the extraction of algorithms from proofs. Here one encounters the same problem of the combination of the Axiom of Choice with classical logic. Up to now there were only two possibilities: (i) to use the bar recursive Dialectica interpretation of the Axiom of Choice due to Spector $[10,22]$; (ii) to avoid the Axiom of Choice whenever possible, for example by encoding functions as relations such as done by Murthy in [17,4].

We improve on (i) since our interpretation is computationally much more direct than Gödel's Dialectica interpretation, and the resulting algorithm is more intuitive than bar recursion.

Received June 25, 1995; revised January 13, 1997. 
We improve on (ii), since encoding functions as relations is often unnatural (see the discussion of Higman's lemma in [17]). Moreover, the encoding relies on the definability of a function value $f(x)$ as the smallest $y$ satisfying $\phi(x, y)$, and this only works well if $y$ ranges over an effective well-ordering such as the natural numbers. The approach here allows to interpret directly the first informal proof of Higman's lemma presented in [17], and applies also to the case where $y$ ranges over an arbitrary simple type over $\mathrm{N}$.

Our paper is organized as follows. In the first section we present the formal system under consideration. In Section 2, we enumerate some known difficulties in combining the Axiom of Choice and the Excluded Middle. They appear in quite different forms in different formalisms: as the impossibility of a recursive realization, as a non-conservativity result, and as a problem with a formulation in sequent calculus. In the next section we present the programming language in which the realizing objects live. The central and longer part is then Section 4, the description of a realizability interpretation, with a precise and detailed proof of correctness. This proof of correctness is non-constructive. (We use an intuitionistic meta-theory throughout this paper, unless explicitely stated otherwise.) We show in the next section that this interpretation can be generalized to the case of the axiom of Dependent Choice, and in this case, reduce intuitionistically its correctness to a principle of bar induction. In Section 6, we shortly review Spector's bar recursive interpretation of Double Negation Shift [22], which suggests a computational content of the negative translation of Axiom of Choice based on Gödel's Dialectica translation. We end by a section with a heuristic explanation of our realizability interpretation, based on a game-theoretical analysis of proofs.

\section{§1. Presentation of $H A^{\omega}$.}

1.1. Types. The types of $H A^{\omega}$ are $N$ and with $\tau, \tau^{\prime}$ also $\tau \rightarrow \tau^{\prime}$. Here and below types will be denoted by lower case greek letters $\tau, \tau^{\prime}, \ldots$

1.2. Terms. The terms of $\mathrm{HA}^{\omega}$ are built from (typed) variables and constants using lambda abstraction and (well typed) application. There are countably many variables $x, y, z, \ldots$ for each type $\tau$. The constants are: $0: \mathrm{N}, \mathrm{s}: \mathrm{N} \rightarrow \mathrm{N}$ and $\mathrm{R}_{\tau}: \tau \rightarrow(\mathrm{N} \rightarrow \tau \rightarrow \tau) \rightarrow \mathrm{N} \rightarrow \tau$ for every type $\tau$. Terms are denoted by $M, M^{\prime}$, $N, \ldots$, and $M: \tau$ expresses that the term $M$ has type $\tau$.

1.3. Formulae. Prime formulae are equations of the form $M=M^{\prime}$, with $M, M^{\prime}$ : $\mathrm{N}$. Higher type equations, say $M=M^{\prime}$ with $M, M^{\prime}: \mathrm{N} \rightarrow \mathrm{N}$, are abbreviations of equations of lowest type, such as $M x=M^{\prime} x$ with $x$ fresh. The set of formulae of $\mathrm{HA}^{\omega}$ is generated in the usual way from the prime formulae by the boolean connectives $\wedge, \Longrightarrow, \perp$ and the quantifiers $\forall, \exists$. We use $\phi, \phi^{\prime}, \ldots$ to denote formulae. We abbreviate $\phi \Longrightarrow \perp$ by $\neg \phi$.

1.4. Theory. The theory $H A^{\omega}$, intuitionistic higher-order arithmetic, is built up from three parts: (i) axioms and rules for first order many-sorted intuitionistic predicate logic; (ii) equality axioms and the axiom schema of induction; (iii) lambda calculus axioms and rules and the defining equations of the constants $\mathrm{R}_{\tau}, \mathrm{R}_{\tau} x y 0=$ $x, \mathrm{R}_{\tau} x y(\mathrm{~s} z)=y z\left(\mathrm{R}_{\tau} x y z\right)$. Thus our theory $\mathrm{HA}^{\omega}$ essentially coincides with $\mathrm{HA}^{\omega}$ from [26], the only difference being that we consider $V$ as defined and use the lambda - version instead of the combinator version. We do not include extensionality axioms 
or rules into the theory $\mathrm{HA}^{\omega}$. The theory $\mathrm{HA}_{c}^{\omega}$ is $\mathrm{HA}^{\omega}$ with classical logic; $\mathrm{HA}_{-}^{\omega}$, minimal higher-order arithmetic, is $\mathrm{HA}^{\omega}$ without the axiom schema $\perp \Longrightarrow \phi$.

1.5. The Axiom of Choice. Theories of classical (intuitionistic) analysis can be obtained from $\mathrm{HA}_{c}^{\omega}\left(\mathrm{HA}^{\omega}\right)$ by adding the Axiom of Choice. The Axiom of Choice of types $\tau, \tau^{\prime}$, denoted by $\mathrm{AC}\left(\tau, \tau^{\prime}\right)$, is the axiom schema

$$
\left[\forall x: \tau \exists y: \tau^{\prime} \phi(x, y)\right] \Longrightarrow \exists f: \tau \rightarrow \tau^{\prime} \forall x: \tau \phi(x, f x)
$$

(schematic in formula $\phi$ ). Here we will mainly consider $\operatorname{AC}(N, \tau)$ (schematic in $\tau$ ), that we may abbreviate to $A C$. The axiom $A C$ is sufficiently strong to formalize a large part of classical analysis. Intuitionistically, $A C$ is not a strong axiom, as may be expected from the Brouwer-Heyting-Kolmogorov interpretation of a $\forall \exists$ prefix. More formally, it follows from results of Goodman [7] that adding $A C(N, N)$ and $A C(N, N \rightarrow N)$ to $H A^{\omega}$ is conservative over Heyting Arithmetic.

1.6. A negative translation. We will use the denotation $\nabla \phi$ for $\neg \neg \phi$. This notation is justified by the fact that $\neg \neg$ can be thought of as a modal operator on formulae; we can prove indeed that $\nabla \phi$ follows from $\phi$, and $\nabla \psi$ from $\nabla \phi$ and $\nabla[\phi \Longrightarrow \psi]$.

As negative translation we use a standard version of the double negation translation, i.e., prefixing prime formulae and $\exists$ by $\nabla$. The idea is essentially due to Kolmogorov [13]. The negative translation of a formula $\phi$, denoted by $\mathrm{K} \phi$, is defined by the six inductive clauses below (throughout this paper, $\equiv$ denotes syntactical identity). The double negation of prime formulae requires some explanation.

Since absurdity will not be interpreted by the empty type, we cannot realize the Ex Falso axiom $\perp \Longrightarrow \phi$ for all $\phi$. However, we must be able to prove $\perp \Longrightarrow \mathrm{K} \phi$ in order to preserve provability. We overcome this problem by exercising some care in the negative translation. We employ the fact that $\perp \Longrightarrow(\phi \Longrightarrow \perp)$ can be proved for all $\phi$ by two simple $\Longrightarrow$-introductions, without using the Ex Falso axiom. Hence it suffices that formulae $\mathrm{K} \phi$ are stable, ${ }^{1}$ in particular the equivalence to a negative formula should be provable without appealing to the Ex Falso axiom. The stability of prime formulae requires the Ex Falso axiom. Therefore the negative translation of a prime formula $\phi$ will be $\nabla \phi$.

- $\mathrm{K} \perp \equiv \perp$

- $\mathrm{K} \phi \equiv \nabla \phi$ if $\phi$ is a prime formula

- $\mathrm{K}[\phi \Longrightarrow \psi] \equiv[\mathrm{K} \phi] \Longrightarrow \mathrm{K} \psi$

- $\mathrm{K}[\phi \wedge \psi] \equiv \mathrm{K} \phi \wedge \mathrm{K} \psi$

- $\mathrm{K} \forall x: \tau \phi \equiv \forall x: \tau \mathrm{K} \phi$

- K $\exists x: \tau \phi \equiv \nabla \exists x: \tau \mathrm{K} \phi$.

The negative translation satisfies the following preservation property: if $\phi$ is provable in $\mathrm{HA}_{c}^{\omega}$, then $\mathrm{K} \phi$ is provable in $\mathrm{HA}_{-}^{\omega}$. In the presence of $\mathrm{AC}$, one cannot expect such a simple preservation result since, as we shall see below, $A C$ is classically much stronger than intuitionistically.

The theory $\mathrm{HA}_{-}^{\omega}+\mathrm{K} \mathrm{AC}$ will be called negative analysis. We need the following technical results.

LEMMA 1.1. The following schemata are provable in $\mathrm{HA}_{-}^{\omega}$ :

(i) $\perp \Longrightarrow \neg \phi$;

\footnotetext{
${ }^{1} \mathrm{~A}$ formula is stable if and only if it is equivalent to the negation of another formula. Equivalently, $\phi$ is stable if and only if $\phi \Longleftrightarrow \nabla \phi$.
} 
(ii) $\nabla \neg \phi \Longleftrightarrow \neg \phi$;

(iii) $\phi \Longrightarrow \nabla \phi$;

(iv) $\mathrm{K} \phi \Longleftrightarrow \nabla \mathrm{K} \phi$;

(v) $\mathrm{K} \exists x: \tau \phi \Longleftrightarrow \nabla \exists x: \tau \phi$, provided $\phi$ is a prime formula;

(vi) $\phi \Longrightarrow \mathrm{K} \phi$, provided $\phi$ is a formula not containing $\Longrightarrow$;

(vii) $\mathrm{K} \neg \phi \Longleftrightarrow \neg \mathrm{K} \phi$.

Proof. The clauses (i)-(iii) and (vi), (vii) are trivial. For (iv), observe that the standard proof of this fact by formula induction does not use the axiom schema $\perp \Longrightarrow \phi$. For all $\phi$ we have $\nabla \exists x: \tau \nabla \phi \Longleftrightarrow \nabla \exists x: \tau \phi$ via $\neg \exists x: \tau \phi \Longleftrightarrow \forall x:$ $\tau \neg \phi$ using (ii), so we have (v).

The negative translation of $A C, K A C$, reads:

$$
[\forall x: \mathrm{N} \nabla \exists y: \tau \mathrm{K} \phi(x, y)] \Longrightarrow \nabla \exists f: \mathrm{N} \rightarrow \tau \forall x: \mathrm{N} \mathrm{K} \phi(x, f x) .
$$

By the stability of formulae after the negative translation, which follows from Lemma 1.1 (iv), $\mathrm{KAC}$ is subsumed by the following axiom schema:

$$
[\forall x: \mathrm{N} \nabla \exists y: \tau \neg \phi(x, y)] \Longrightarrow \nabla \exists f: \mathrm{N} \rightarrow \tau \forall x: \mathrm{N} \neg \phi(x, f x) .
$$

Par abus de langage we will from now on denote this schema by $\mathrm{K} \mathrm{AC}$.

We can now extend the preservation property above to: if $\phi$ is provable in classical analysis, then $\mathrm{K} \phi$ is provable in negative analysis. The proof goes by inspection of the standard preservation proof, taking care that $\perp \Longrightarrow \phi$ is avoided using the lemma above.

§2. Classical logic and the Axiom of Choice. In this section, we enumerate some known difficulties in giving a constructive interpretation of the Axiom of Choice in the presence of classical logic. The main remark is that $K A C$ fails to be an intuitionistic consequence of the Axiom of Choice. ${ }^{2}$ We point out three independent reasons:

- $\mathrm{K} \mathrm{AC}$ makes problematic the constructive interpretation of the notion of function; actually, it refutes the intuitionistic version of Church's Thesis;

- $\mathrm{KAC}$ is proof theoretically very strong, we can actually interpret by $\mathrm{KAC}$ impredicative second-order comprehension, so that one cannot give any predicative interpretation of $\mathrm{K} \mathrm{AC}$;

- a standard computational interpretation of classical arithmetic, the one using infinitary propositional calculus $[18,23]$, when extended to quantification over functions, fails to interpret the Axiom of Choice.

2.1. The impossibility of a recursive realization. In the classical system $H A_{c}^{\omega}+A C$, we may define the characteristic function of any predicate. Indeed, for any statement $\phi(x)$, we have

$$
\exists f \forall x[\phi(x) \Longleftrightarrow[f(x)=0]]
$$

since, by the Excluded Middle,

$$
\forall x \exists y[\phi(x) \Longleftrightarrow[y=0]] .
$$

\footnotetext{
${ }^{2}$ This is to be contrasted with the induction schema over integers, whose negative translation is an instance of the induction schema itself.
} 
If we take for $\phi$ some non-decidable formula, then we may prove in this way the existence of non-recursive functions in $\mathrm{HA}_{c}^{\omega}+\mathrm{AC}$. For example, take $\phi(x) \equiv$ $\forall z \neg T(x, x, z)$, where $T(e, x, y)$ is the standard Kleene predicate (which can be formulated as an equation), i.e., $\phi(x)$ is the (non-)Halting Problem. As a consequence of the above observation we can prove in $\mathrm{HA}_{-}^{\omega}+\mathrm{KAC}$

$$
\nabla \exists f \forall x[[\nabla f(x)=0] \Longleftrightarrow \forall z \neg T(x, x, z)] .
$$

In usual realizability notions $\perp$ has no realizers. As a consequence, $\phi$ is realizable if and only if $\neg \phi$ is not realizable, so $\phi$ is realizable if and only if $\nabla \phi$ is realizable. It follows that there is no recursive realization of the above theorem (1) of $\mathrm{HA}_{-}^{\omega}+\mathrm{KAC}$, and hence there is no sound recursive realization of $\mathrm{HA}_{-}^{\omega}+\mathrm{KAC}$ itself.

For exactly this reason, and because the semantics of the system NuPrl is based on recursive realizability, the work $[17,4]$ restricts itself to a fragment of classical logic that does not include the Axiom of Choice. This often forces one to encode functions as relations, and this encoding may be unnatural (see the discussion of Higman's Lemma in [17]), or even impossible.

It is interesting to analyse in what way our interpretation avoids this difficulty. The main obstacle for recursive realizability is the equivalence between the realizability of $\phi$ and the realizability of $\nabla \phi$, which comes from the fact that the absurd proposition has no realizers. Our interpretation introduces potential "realizers" for the absurd proposition, and because of this, $\nabla \phi$ can be realizable though $\phi$ is not realizable. What is hard to understand intuitively is why taking into account possible "absurd situations" (that will actually never happen) has such an effect.

It also follows from the observations above that in $\mathrm{HA}_{c}^{\omega}+\mathrm{AC}$ we can refute the formula

$$
\mathrm{CT} \equiv \forall f: \mathrm{N} \rightarrow \mathrm{N} \exists e: \mathrm{N} \forall x: \mathrm{N} \exists y: \mathrm{N}[T(e, x, y) \wedge U(y)=f(x)]
$$

where $T(e, x, y)$ is again the standard Kleene predicate and $U$ is Kleene's result extracting function, i.e., $U(y)=z$ means that the output of the computation with code $y$ is $z$. This formula CT expresses Church's Thesis in an intuitionistic way, stating that every function $f$ has a recursive index $e$.

Note that both $\mathrm{HA}_{c}^{\omega}+\mathrm{CT}$ and $\mathrm{HA}^{\omega}+\mathrm{AC}+\mathrm{CT}$ are consistent due to the existence of models in which all functions are recursive, such as HEO, see [26]. Since $C T$ is a formula not containing $\Longrightarrow$, it implies its negative translation $K C T$ by Lemma 1.1(vi). We can thus prove $\perp$ in the system $H A_{-}^{\omega}+K A C+C T$.

It may come as a surprise that we come up with a recursive object that realizes a principle which itself refutes CT. This, however, has nothing to do with adopting realizers of $\perp$, but with the extensionality of our realizing objects. Note that CT expresses recursiveness in an intensional way, by stating that every function $f$ has an index $e$. In this respect we cite Troelstra [27], who credits Kreisel [14] for the essence of the argument: 'choice, recursiveness and extensionality are incompatible relative to intuitionistic finite-type arithmetic'. Our realizing objects are extensional, and hence they do not realize $C T$. As a consequence, $\neg C T$ is trivially realized.

A particularly simple proof can be obtained by employing the fact that our realizing objects are not only extensional, but even continuous. Assume by contradiction that $M$ is a continuous realizer of CT. Let $e=M(\lambda x .0)$, that is, 
$e$ is an index of the zero function. Let $l$ be the length of the initial segment of the zero function such that $M$ is constant on the corresponding open set in the Baire topology. This open set contains a function, say $f$, which is not the zero function. We have $M f=e$, so $e$ is also an index of $f$, contradiction.

2.2. Proof theoretic strength of $K A C$. A second difficulty in interpreting $K A C$ comes from the fact that in intuitionistic logic, $K A C$ is proof-theoretically much stronger than $A C$.

We have seen already that $H A^{\omega}+A C$ is conservative over $H A$ [7]. In contrast, by using the fact that in $H A_{c}^{\omega}+A C$ we may define the characteristic function of any predicate, it is not difficult to see that we can interpret the second-order Comprehension Axiom in $\mathrm{HA}_{-}^{\omega}+\mathrm{KAC}$. This system is proof theoretically much stronger than HA (see, for instance, [21], Section 8.5), which implies that there cannot be any predicative way of modeling $\mathrm{HA}_{-}^{\omega}+\mathrm{KAC}$.

These remarks may explain a comment of Gödel after presenting the negative translation [6]: "Intuitionism appears to introduce genuine restrictions only for analysis and set theory; these restrictions, however, are due to the rejection, not of the principle of Excluded Middle, but of notions introduced by impredicative definitions."

It can be shown that the negative translation extends to impredicative calculi, and by Gödel's own interpretation of the Axiom of Choice by constructible sets, it is even possible to make sense in this way of the full higher-order arithmetic with the Excluded Middle and the Axiom of Choice in an "intuitionistic" impredicative higher-order arithmetic. (See the introductory note to $1933 e$ in [6]).

An important aspect that is not covered in this comment however is a more "intensional" aspect of logical systems, independent of their proof-theoretic strength. From an intuitionistic viewpoint, the proof object, that counts as evidence for the truth of a proposition, is of primary importance.

2.3. Problem in sequent calculus. Let us analyse now another way to get a possible computational content from classical arguments by considering Gentzen's sequent calculus formulation of classical arithmetic. One elegant approach is to use derivations in infinitary propositional logic $[18,23]$. There is a general cut-elimination result for this logic which gives a computational interpretation of proofs. If we introduce countable disjunction and conjunction, we can interpret in this way classical quantification over natural numbers and give a computational interpretation of proofs in classical arithmetic.

A natural attempt is then to introduce disjunction and conjunction over the set of all number theoretic functions, and hope that we can interpret in this way classical quantification over number theoretic functions. Then, as noticed in Tait's paper [23], there is a natural formulation of the Axiom of Choice in infinitary propositional logic. We start from atoms $\phi_{m n}, \overline{\phi_{m n}}$, and axioms

$$
\Gamma, \phi_{m n}, \overline{\phi_{m n}}
$$

and we ask whether it is possible to build a cut-free proof, uniformly in $\phi$, of

$$
\bigvee_{m} \bigwedge_{n} \overline{\phi_{m n}}, \bigvee_{f} \bigwedge_{m} \phi_{m f(m)}
$$


from these axioms. It is not difficult to see that there cannot be such a cut-free proof. We will explain this in some detail. ${ }^{3}$ Assume by contradiction $\Delta$ is a cut-free proof of (2).

First, observe that all conjunctions in (2) are countable, so by the subformula property all conjunctions in $\Delta$ are countable, so that $\Delta$ is at most countably branching. Since $\Delta$ is also well founded, it follows that $\Delta$ is countable and that at most countably many functions $f_{0}, f_{1}, \ldots$ appear in $\Delta$.

Second, replace every subformula of the form $\bigvee_{f} \phi(f)$ in $\Delta$ by $\bigvee_{k} \phi\left(f_{k}\right)$ and call the resulting tree $\Delta^{\prime}$. Observe that $\bigvee_{f} \phi(f)$ can only be introduced in $\Delta$ from some $\phi\left(f_{k}\right)$. It follows that $\Delta^{\prime}$ is indeed a proof of

$$
\bigvee_{m} \bigwedge_{n} \overline{\phi_{m n}}, \bigvee_{k} \bigwedge_{m} \phi_{m f_{k}(m)}
$$

Third, the above conclusion of $\Delta^{\prime}$ is false. This can be seen by taking $f$ to be a function diagonalizing over $f_{0}, f_{1}, \ldots$, e.g. $f(n)=f_{n}(n)+1$, and $\phi_{m n} \Longleftrightarrow$ $f(m)=n$. Indeed, $\bigvee_{m} \bigwedge_{n} \overline{\phi_{m n}}$ is false since $f$ is total, and $\bigvee_{k} \wedge_{m} \phi_{m f_{k}(m)}$ is false since $f$ is by construction different from all $f_{k}$ s.

§3. The programming language $\mathscr{P}$. The programming language $\mathscr{P}$ extends the types, terms and equations of $\mathrm{HA}^{\omega}$ with type constants for interpreting $\perp$ and equations, type constructors for lists and pairs, term constants associated to the new types, general recursion and infinite terms to form choice sequences.

3.1. Types of $\mathscr{P}$. The types of $\mathscr{P}$ are $\mathrm{N}$, Unit, Abs and with $\tau, \tau^{\prime}$ also $\tau \rightarrow \tau^{\prime}$, $\tau \times \tau^{\prime}$ (cartesian product) and [ $\left.\tau\right]$ (lists over type $\tau$ ). The type Unit serves to interpret prime formulae, and the type Abs to interpret $\perp$. The type Abs will not be empty. Like in the case of $\mathrm{HA}^{\omega}$, types will be denoted by lower case greek letters $\tau, \tau^{\prime}, \ldots$ The types of $\mathrm{HA}^{\omega}$ will be called $\mathrm{N}$-types.

3.2. Terms of $\mathscr{P}$. The terms of $\mathscr{P}$ are built from (typed) variables and constants using lambda abstraction, (well typed) application, and the formation of infinite terms: if $M_{0}, M_{1}, \ldots$ is an infinite sequence of terms of type $\tau$, then $\left(\not x x . M_{x}\right)$ is a term of type $\mathrm{N} \rightarrow \tau$. (The infinite terms are not for computational purposes, they only play a role in the termination proof.) There are countably many variables $x, y, z, \ldots$ for each type $\tau$. The set of constants of $\mathscr{P}$ extends that of $\mathrm{HA}^{\omega}$ with constants $\mathrm{R}_{\tau}$ for types $\tau$ that are not $\mathrm{N}$-types, () : Unit, Dummy : Abs, Axiom $_{1}$, Axiom $_{2}: \mathrm{N} \rightarrow$ Abs, constants for general recursion (fixpoint combinators of all appropriate types) and constants for pairing and projection and list construction and destruction. Terms are again denoted by $M, M^{\prime}, N, \ldots$, and $M: \tau$ expresses that the term $M$ has type $\tau$.

3.3. Equations of $\mathscr{P}$. The only formulae of $\mathscr{P}$ are equations of the form $M=M^{\prime}$, with $M, M^{\prime}$ terms of $\mathscr{P}$ of the same type, not necessarily N.

3.3.1. Theory of $\mathscr{P}$. The theory of $\mathscr{P}$ is equational, built up from the usual lambda calculus axioms and rules, defining equations for the constants $\mathrm{R}_{\tau}$ as in $\mathrm{HA}^{\omega}$, but now for all types $\tau$ of $\mathscr{P}$, pairing axioms and list axioms and axioms for

\footnotetext{
${ }^{3} \mathrm{Cf}$. [23], Tait describes this result as known already, but does not give further references. We think Tait was implicitely referring to the failure of completeness for uncountable infinitary logic, see for instance [20].
} 
general recursion as usual, and the following axiom schema for infinite terms:

$$
(\beta \beta) \quad\left(\not \lambda x \cdot M_{x}\right) \underline{k}=M_{k}
$$

(schematic in $\tau, M_{0}, M_{1}, \ldots$ of type $\tau$ and natural number $k$ ).

3.3.2. Pragmatics of $\mathscr{P}$. We allow ourselves a liberal use of $\mathscr{P}$. We will assume that all terms are well typed and we will reduce type information to a minimum that is required to reconstruct the type of a well typed term from the context. For every natural number $k$, we abbreviate $\mathrm{s}(\ldots(\mathrm{s} 0) \ldots$ ) (with $k$ times $\mathrm{s}$ ) by $\underline{k}$, called the numeral $\underline{k}$. The term $\underline{k}_{\tau}$ of N-type $\tau$ is defined inductively by: $\underline{k}_{\mathrm{N}} \equiv \underline{k}$; $\underline{k}_{\tau \rightarrow \tau^{\prime}} \equiv \lambda x: \tau . \underline{k}_{\tau^{\prime}}$. We write pairs as $(M, N)$ and triples as $(M, N, P)$ instead of $(M,(N, P))$. We even write $\lambda(x, y) . M$ instead of $\lambda z: \tau \times \tau^{\prime} . M^{\prime}$, where $M^{\prime}$ is obtained from $M$ by replacing $x$ by the first projection of $z$ and $y$ by the second. Lists are denoted by $\left[M_{1}, \ldots, M_{n}\right]\left(M_{i}: \tau\right.$ for $\left.1 \leq i \leq n\right)$. Adding a term $H$ at the beginning of a list $L$ will be denoted by $H: L$.

Instead of the explicit use of fixpoint combinators we define terms by giving the recursion equations. As an example we define a term get : $\mathrm{N} \rightarrow\left[\mathrm{N} \times \tau \times \tau^{\prime}\right] \rightarrow(\tau \rightarrow$ $\left.\tau^{\prime} \rightarrow \tau^{\prime \prime}\right) \rightarrow \tau^{\prime \prime} \rightarrow \tau^{\prime \prime}$, which will play a role in the sequel. The term (get $x l f a$ ) searches the list $l$ for the first triple whose first component matches $x$; if such a triple is found, then the output is $f$ applied to the second and third component of the triple, otherwise the output is $a$. Formally,

$$
\begin{aligned}
\text { get } x[] f a & =a \\
\text { get } x\left(\left(x^{\prime}, y, y^{\prime}\right): l\right) f a & \left.=\text { if }\left(x=x^{\prime}\right) \text { then }\left(f y y^{\prime}\right) \text { else (get } x l f a\right) .
\end{aligned}
$$

Here and below, if $\left(M=M^{\prime}\right)$ then ... else... (with $M, M^{\prime}$ of type $\mathrm{N}$ ) is a sugared version of a well-known primitive recursive term.

3.4. Known facts about $\mathscr{P}$. There exists a reduction relation $\longrightarrow$ on the terms of $\mathscr{P}$ such that the reflexive, symmetric and transitive closure of $\longrightarrow$ coincides with $=$ (convertibility) on the terms of $\mathscr{P}$. Moreover, $\longrightarrow$ satisfies:

(i) the Church Rosser Theorem;

(ii) every closed normal form of type $\mathrm{N}$ is a numeral $\underline{k}$;

(iii) every closed normal form of type Unit is ();

(iv) every closed normal form of type Abs is either Dummy or of the form Axiom $1 \underline{k}$ or Axiom $_{2} \underline{k}$;

(v) the Continuity Lemma: let $M:(\mathrm{N} \rightarrow \tau) \rightarrow \tau^{\prime}$ and $N: \mathrm{N} \rightarrow \tau$ be such that $M N$ has a closed normal form. Then there exists a natural number $m$ such that for all $N^{\prime}: N \rightarrow \tau$ with $N \underline{i}=N^{\prime} \underline{i}$ for all $i<m$ we have $M N=M N^{\prime}$. In particular we have extensionality: if $N \underline{i}=N^{\prime} \underline{i}$ for all natural numbers $i$, then $M N=M N^{\prime}$.

§4. A realizability interpretation. Realizability, due to Kleene, aims at formalizing the notion of constructive truth, see [25] for an overview. A realizability interpretation interprets a logic, usually an extension of Heyting Arithmetic, in a programming language. More specifically, to each formula $\phi$ of the logic is associated a type $|\phi|$ of the programming language. One then defines by formula induction when a program of type $|\phi|$ realizes the formula $\phi$. Intuitively, it means that this program is a constructive justification of the formula $\phi$. To each proof of a closed formula $\phi$ is associated a program of type $|\phi|$ which realizes the formula $\phi$. 
In this section a realizability interpretation of $\mathrm{HA}_{-}^{\omega}+\mathrm{KAC}$ in $\mathscr{P}$ will be given, essentially modified realizability due to Kreisel [14], together with realizers of $\perp$. It consists of a mapping of formulas of $\mathrm{HA}^{\omega}$ to types of $\mathscr{P}$ together with a realizability relation between programs in $\mathscr{P}$ and formulas of $H A^{\omega}$, where the program has the type to which the formula is mapped. The main result will be that every theorem of $\mathrm{HA}_{-}^{\omega}+\mathrm{K}$ AC can be realized in $\mathscr{P}$. The difficult step in proving this result is the realization of $\mathrm{K} \mathrm{AC}$.

4.1. Mapping formulas of $H A^{\omega}$ to types of $\mathscr{P}$. The idea behind this mapping is usually referred to as "forgetting dependencies". In an uncurried form it is already present in [14]. By formula induction we define a type $|\phi|$ of $\mathscr{P}$ for every formula $\phi$ of $\mathrm{HA}^{\omega}$ :

- $\left|M=M^{\prime}\right| \equiv$ Unit

- $|\perp| \equiv$ Abs

- $|\phi \Longrightarrow \psi| \equiv|\phi| \rightarrow|\psi|$

- $|\phi \wedge \psi| \equiv|\phi| \times|\psi|$

$\bullet|\forall x: \tau \phi| \equiv \tau \rightarrow|\phi|$

- $|\exists x: \tau \phi| \equiv \tau \times|\phi|$.

Note that the domains of quantification in $\mathrm{HA}^{\omega}$ are types of $\mathrm{HA}^{\omega}$, and hence of $\mathscr{P}$, so that the mapping || is well defined.

4.2. Reducibility in $\mathscr{P}$. In order to define the realizability relation we need a notion of reducibility for closed terms of $\mathscr{P}$ of $\mathrm{N}$-type. By induction on the $\mathrm{N}$-type we define:

- $M: \mathrm{N}$ is reducible if and only if $M$ reduces to a numeral.

- $M: \tau \rightarrow \tau^{\prime}$ is reducible if and only if $M N$ is reducible for every reducible $N: \tau$. In the sequel, we shall need the following technicalities.

DEFINITION 4.1. Two expressions $E$ and $E^{\prime}$ (terms or formulae) are called related if they are syntactically identical up to the indices of the constants Axiom ${ }_{i}$. Note that related terms are of the same type.

LEMMA 4.2. If $M$ and $M^{\prime}$ are two related terms of type $\mathrm{N}$, then $M=\underline{n}$ if and only if $M^{\prime}=\underline{n}$. If $M$ and $M^{\prime}$ are two related terms of type Unit, then $M=()$ if and only if $M^{\prime}=()$.

Proof. By induction on the length of reduction sequences.

LEMMA 4.3. If $M$ and $M^{\prime}$ are two related terms of $\mathrm{N}$-type, then $M$ is reducible if and only if $M^{\prime}$ is reducible.

Proof. By an easy induction on the common N-type of $M$ and $M^{\prime}$, using Lemma 4.2 for the base case $N$.

4.3. Realizability relation. We give an inductive definition of " $M$ realizes $\phi$ ", where $\phi$ is a closed formula of $\mathrm{HA}^{\omega}$ with possibly closed reducible terms of $\mathscr{P}$ occurring in the prime constituents of $\phi$, and $M$ a closed term of $\mathscr{P}$ of type $|\phi|$.

- $M$ : Abs realizes $\perp$ if and only if $M=$ Axiom $_{i} \underline{k}$ for some $i=1,2$ and numeral $\underline{k}$.

- $M$ : Unit realizes $M_{1}=M_{2}$ if and only if $M=()$ and $M_{1}$ and $M_{2}$ reduce in $\mathscr{P}$ to the same numeral. 
- $M:|\phi| \rightarrow|\psi|$ realizes $\phi \Longrightarrow \psi$ if and only if $M N$ realizes $\psi$ whenever $N$ realizes $\phi$.

- $M:|\phi \wedge \psi|$ realizes $\phi \wedge \psi$ if and only if $M=(N, P)$ with $N$ realizes $\phi$ and $P$ realizes $\psi$.

- $M: \tau \rightarrow|\phi|$ realizes $\forall x: \tau \phi$ if and only if $M N$ realizes $\phi[x:=N]$ for all reducible $N: \tau$.

- $M: \tau \times|\phi|$ realizes $\exists x: \tau \phi$ if and only if $M=(N, P)$ with $N: \tau$ reducible and $P$ realizes $\phi[x:=N]$.

Note that the above definition uses reducibility for $\mathrm{N}$-types only. In the case $M_{1}=$ $M_{2}$ above, the equation is of type N. The terms $M_{1}$ and $M_{2}$ come from (possibly) open terms of $\mathrm{HA}^{\omega}$ in which closed reducible terms of $\mathscr{P}$ are substituted for the variables. Thus $M_{1}$ and $M_{2}$ are closed and reducible, since all constants of $\mathrm{HA}^{\omega}$ are reducible constants of $\mathscr{P}$. Hence we can verify $M_{1}=M_{2}$ in $\mathscr{P}$, relying on Fact (i) and (ii) from 3.4.

In the sequel, we shall need the following technical lemma.

LEMMA 4.4. If $\phi$ and $\phi^{\prime}$ are two related formulae, and $M, M^{\prime}$ two related terms, then $M$ realizes $\phi$ if and only if $M^{\prime}$ realizes $\phi^{\prime}$.

Proof. By an easy induction on the realization relation, using Lemma 4.2 and Lemma 4.3.

4.4. Main result and applications. In this subsection we formulate the main result, sketch a proof and give two applications of the main result. The essential and difficult step in the proof of the main result, the realization of $K A C$ is given in the next subsection.

THEOREM 4.5. Every theorem of $\mathrm{HA}_{-}^{\omega}+\mathrm{K} \mathrm{AC}$ can be realized by a term in $\mathscr{P}$ in which no infinite terms and no constants Axiom $_{i}$ occur $(i=1,2)$.

Proof. Apart from the realization of $\mathrm{K} \mathrm{AC}$, the proof is more or less standard. For example, the axiom $\forall x: \mathrm{N} \neg(\mathrm{s} x=0)$ is realized by $M \equiv \lambda x: \mathrm{N} \lambda h$ : Unit.Dummy. Indeed, $M \underline{n}$ realizes $\neg(s \underline{n}=0)$ for every natural number $n$, since nothing realizes $\underline{s} \underline{n}=0$ (here we use Fact (i) and (ii) from 3.4).

4.4.1. Application 1: the consistency of analysis. The main result immediately implies the consistency of analysis. Assume $\perp$ is provable in $\mathrm{HA}_{c}^{\omega}+\mathrm{AC}$. Then $\mathrm{K} \perp$, i.e., $\perp$, is provable in $H A_{-}^{\omega}+\mathrm{KAC}$. Hence $\perp$ is realizable by a term of $\mathscr{P}$ in which no constants $A_{x i o m}$ occur $(i=1,2)$. This is impossible by the definition of realization.

4.4.2. Application 2: how to compute witnesses with $A C$ and classical logic. Assume a formula $\phi(x)$ of $\mathrm{HA}^{\omega}$, with $x: \mathrm{N}$, is decidable, i.e., of the form $M_{\phi} x=0$ for suitable closed term $M_{\phi}$ of $H^{\omega}$. We will freely write $\phi(x)$ instead of $M_{\phi} x=0$. Assume $\exists x: \mathrm{N} \phi(x)$ is a theorem of $\mathrm{HA}_{c}^{\omega}+\mathrm{AC}$. Then $\nabla \exists x: \mathrm{NK} \phi(x)$ is a theorem of $\mathrm{HA}_{-}^{\omega}+\mathrm{KAC}$. By Lemma $1.1(\mathrm{v})$ we have that $\nabla \exists x: \mathrm{N} \phi(x)$ is a theorem of $\mathrm{HA}_{-}^{\omega}+\mathrm{KAC}$, and hence realizable by a term not containing constants Axiom $_{i}$ $(i=1,2)$, say by $M$. We have that

$$
N \equiv \lambda(x, h): \mathrm{N} \times \text { Unit.if } \phi(x) \text { then }\left(\text { Axiom }_{1} x\right) \text { else Dummy }
$$


realizes $\neg \exists x: N \phi(x)$. So $M N$ realizes $\perp$ and must hence be convertible to Axiom $\underline{n}$ for some numeral $\underline{n}$. We claim $\phi(\underline{n})$, i.e., $\underline{n}$ is a witness. Consider the following extensionally equal terms:

$$
\begin{gathered}
F \equiv \lambda x: \mathrm{N} \lambda h: \text { Unit.if } \phi(x) \text { then (Axiom } 1 x) \text { else Dummy } \\
\left.F^{\prime} \equiv \lambda x: \mathrm{N} \lambda h: \text { Unit.if } \phi(x) \text { then (if } \phi(x) \text { then (Axiom } 1 x\right) \text { else Dummy) } \\
\text { else Dummy. }
\end{gathered}
$$

We have $N=\lambda(x, h) . F x h$. Furthermore, $F^{\prime}$ can be obtained from $F$ by replacing Axiom ${ }_{1}$ by $\lambda x$.if $\phi(x)$ then (Axiom $1 x$ ) else Dummy. Let us call this substitution $\sigma$, and let $E \sigma$ denote the result of applying the substitution $\sigma$ to the expression $E$, for any expression $E$.

Since $F$ and $F^{\prime}$ are extensionally equal we have by extensionality:

$$
\text { Axiom }_{1} \underline{n}=M N=M(\lambda(x, h) . F x h)=M\left(\lambda(x, h) \cdot F^{\prime} x h\right) .
$$

We have $F \sigma=F^{\prime}$ and, since $M$ does not contain the constant Axiom $1, M \sigma \equiv M$. Hence we can calculate:

$$
\left(\text { Axiom }_{1} \underline{n}\right) \sigma=(M(\lambda(x, h) . F x h)) \sigma=M\left(\lambda(x, h) \cdot F^{\prime} x h\right)=\text { Axiom } \underline{\underline{n}} .
$$

It follows that

$$
\text { Axiom }_{1} \underline{n}=\text { if } \phi(\underline{n}) \text { then }\left(\text { Axiom }_{1} \underline{n}\right) \text { else Dummy. }
$$

Using Fact (iv) from 3.4 we get $\phi(\underline{n})$.

4.4.3. Application 3: extraction of algorithms. Let $\phi(y, x)$ be $M_{\phi} y x=0$ with $x: \mathrm{N}$ and $y: \tau$. Assume $\forall y: \tau \exists x: \mathrm{N} \phi(y, x)$ is a theorem of $\mathrm{HA}_{c}^{\omega}+\mathrm{AC}$. Like in

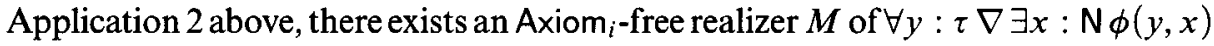

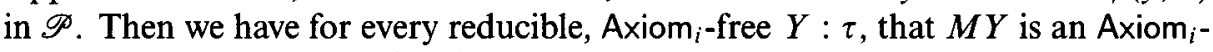
free realizer of $\nabla \exists x: N \phi(Y, x)$. Define

$$
N \equiv \lambda y \lambda(x, h): \mathrm{N} \times \text { Unit.if } \phi(y, x) \text { then }\left(\text { Axiom }_{1} x\right) \text { else Dummy, }
$$

then $N Y$ realizes $\neg \exists x: \mathrm{N} \phi(Y, x)$ as above. Hence $M Y(N Y)$ realizes $\perp$ and hence reduces to $A_{x i o m} \underline{n}$ for some numeral $\underline{n}$. Like above, we have $\phi(Y, \underline{n})$. At this point, observe that in the reduction of $M Y(N Y)$ to Axiom $\underline{n}$ no special features of the constants Abs, Axiom $i$, Dummy are used. As a consequence, they may be considered as variables as well. Let $\sigma$ be the substitution [Abs $:=\mathrm{N}$, Axiom $1:=\lambda x$ : N. $x$, Dummy $:=0$ ]. Then $(M Y(N Y)) \sigma$ is a well-typed term which reduces to $\underline{n}$. Thus the term $F \equiv(\lambda y: \tau \cdot M y(N y)) \sigma$ is a well-typed term of $\mathscr{P}$ with the property

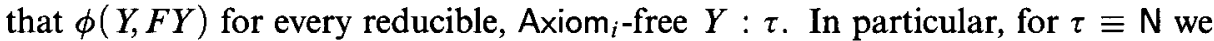
have $\phi(\underline{n}, F \underline{n})$ for every numeral $\underline{n}$.

4.5. Realization of $K A C$. Recall that $K A C$ is the following schema:

$$
[\forall x: \mathrm{N} \nabla \exists y: \tau \neg \phi(x, y)] \Longrightarrow \nabla \exists f: \mathrm{N} \rightarrow \tau \forall x: \mathrm{N} \neg \phi(x, f x) \text {. }
$$

We start with some preliminary calculations:

$$
\begin{aligned}
|\forall x: \mathrm{N} \nabla \exists y: \tau \neg \phi(x, y)| & \equiv \mathrm{N} \rightarrow((\tau \times(|\phi| \rightarrow \text { Abs })) \rightarrow \text { Abs }) \rightarrow \text { Abs } \\
|\neg \exists f: \mathrm{N} \rightarrow \tau \forall x: \mathrm{N} \neg \phi(x, f x)| & \equiv((\mathrm{N} \rightarrow \tau) \times(\mathrm{N} \rightarrow|\phi| \rightarrow \text { Abs })) \rightarrow \text { Abs. }
\end{aligned}
$$

A realizer of $\mathrm{K} \mathrm{AC}$ should be a term $M$ such that $M H P$ realizes $\perp$ whenever $H$ realizes $\forall x: \mathrm{N} \nabla \exists y: \tau \neg \phi(x, y)$ and $P$ realizes $\neg \exists f: \mathrm{N} \rightarrow \tau \forall x: \mathrm{N} \neg \phi(x, f x)$. Moreover, $M$ should not contain constants $\operatorname{Axiom}_{i}(i=1,2)$. The general idea is 
to approximate a function witnessing $\exists f: \mathrm{N} \rightarrow \tau \forall x: \mathrm{N} \neg \phi(x, f x)$ by means of a list $L$ of triples $(X, Y, Z)$, where $X: N$ and $Y: \tau$ are reducible, and $Z$ realizes $\neg \phi(X, Y)$. Given such a list $L=\left[\left(X_{1}, Y_{1}, Z_{1}\right), \ldots,\left(X_{n}, Y_{n}, Z_{n}\right)\right]$ with all the $X_{i}$ s distinct, we consider a function fun $L: \mathrm{N} \rightarrow \tau$ which maps $X_{i}$ to $Y_{i}(1 \leq i \leq n)$ and takes function values $\underline{0}_{\tau}$ in arguments different from all $X_{i}$ s. Formally:

$$
\text { fun } l x=\text { get } x l(\lambda y: \tau \lambda z:|\phi| \rightarrow \text { Abs. } y) \underline{0}_{\tau} \text {. }
$$

Furthermore, we consider a function $\lambda x:$ N.rea $L x A: \mathrm{N} \rightarrow|\phi| \rightarrow$ Abs which maps $X_{i}$ to the realizer $Z_{i}(1 \leq i \leq n)$ and takes values $A$ (to be specified below) in arguments different from all $X_{i}$ s. Formally:

$$
\text { rea } l x a=\text { get } x l(\lambda y: \tau \lambda z:|\phi| \rightarrow \text { Abs.z) } a \text {. }
$$

Consider

$$
P(\text { fun } L, \lambda x: \text { N.rea } L x A) \text {. }
$$

If $\lambda x:$ N.rea $L x A$ realizes $\forall x \neg \phi(x$, fun $L x)$, then we would have that $P$ (fun $L, \lambda x$ : N.rea $L \times A$ ) realizes $\perp$ and we would be done. However, this is in general not the case since we cannot choose $A$ such that $A$ realizes $\neg \phi\left(x, \underline{0}_{\tau}\right)$ for all $x$ different from all $X_{i}$ s. We claim that, as $A$ may depend on $x$, there is a possibility to construct $A$ in such a way that it allows us to compute a better approximation of the function witnessing $\exists f: \mathrm{N} \rightarrow \tau \forall x: \mathrm{N} \neg \phi(x, f x)$. The type of $A$ must be $|\phi| \rightarrow$ Abs, so we must have

$$
A \equiv \lambda x^{\prime}:|\phi| \cdot \cdots,
$$

where $\cdots$ is of type Abs. It is tempting to fill in $\cdots$ with $\left(\right.$ Axiom $\left._{i} x\right)$. The resulting term

$$
P\left(\text { fun } L, \lambda x \text {.rea } L x\left(\lambda x^{\prime}:|\phi| \cdot \operatorname{Axiom}_{i} x\right)\right)
$$

of type Abs is not a solution, since it contains Axiom ${ }_{i}$, but it will play an important role in the discussion below. Note that $\lambda x$ : N.rea $L x A$ only accesses $A$ in case $x$ does not occur as first component of a triple in $L$. The basic intuition is that, if the above term reduces to Axiom $i \underline{k}$, then this tells us that $P$ needs more information about its arguments, in particular it needs a function value and a realizer for the argument $k$.

Observe that, so far, $H$ realizing $\forall x: N \nabla \exists y: \tau \neg \phi(x, y)$ has not been used. For filling in $\cdots$ we use $H$. Recall that the type of $H$ is $\mathrm{N} \rightarrow((\tau \times(|\phi| \rightarrow$ Abs $)) \rightarrow$ Abs $\rightarrow$ Abs. The obvious way to continue is putting

$$
A \equiv \lambda x^{\prime}:|\phi| . H x \cdots \text {. }
$$

Now $\cdots$ is of type $(\tau \times(|\phi| \rightarrow$ Abs $)) \rightarrow$ Abs and is hence of the form $\lambda(y, z) . \cdots$, so that we have

$$
A \equiv \lambda x^{\prime}:|\phi| . H x(\lambda(y, z) . \cdots)
$$

where $\cdots$ is again of type Abs. The crucial idea is now to put

$$
A \equiv \lambda x^{\prime}:|\phi| . H x(\lambda(y, z) \cdots((x, y, z): L))
$$

where $\cdots$ stands for a recursive call of the whole procedure described above.

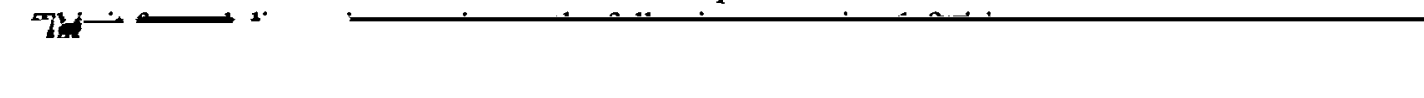


Here (member $x L$ ) tests if $x$ occurs as first component of a triple in $L$. Since the only occurrence of Axiom in $_{F}$ is the one which is explicitly shown, we have that

$$
F^{\prime}=F\left[\text { Axiom }_{1}:=\lambda x \text {.if }(\text { member } x L) \text { then Dummy else }\left(\text { Axiom }_{\downarrow} x\right)\right] \text {. }
$$

Recall that $P$ (fun $L, F)=\Phi^{\prime} P H L=$ Axiom $_{1} \underline{k}$. By extensionality we have $P\left(\right.$ fun $\left.L, F^{\prime}\right)=P($ fun $L, F)=$ Axiom $_{1} \underline{k}$. By substitution we get

$$
\begin{aligned}
& P\left(\text { fun } L, F^{\prime}\right)=\left(\text { Axiom }_{1} \underline{k}\right)\left[\text { Axiom }_{1}:=\lambda x \text {.if }(\operatorname{member} x L)\right. \text { then Dummy } \\
& \text { else } \left.\left(\text { Axiom }_{1} x\right)\right] \\
& =\text { if }(\text { member } \underline{k} L) \text { then Dummy else }\left(\text { Axiom }_{1} \underline{k}\right) .
\end{aligned}
$$

By Fact (i) (Church Rosser Theorem) from 3.4 we get $\neg$ (member $\underline{k} L$ ). This is (ii) and completes the proof of the lemma.

Using the lemma above we can prove by contradiction that $\Phi P H$ [] realizes $\perp$. We give an informal argument, which can easily be formalized using the axiom of Dependent Choice and classical logic. The argument is similar to the argument used by Tait in [24]. Suppose $\Phi P H$ [] does not realize $\perp$. Then, by the lemma above, there exist $X_{1}, Y_{1}, Z_{1}$ such that $\left[\left(X_{1}, Y_{1}, Z_{1}\right)\right]$ satisfies the conditions of the lemma, in particular $\Phi P H\left[\left(X_{1}, Y_{1}, Z_{1}\right)\right]$ does not realize $\perp$. Applying the lemma again yields $X_{2}, Y_{2}, Z_{2}$ such that ... Iterating this argument infinitely many times yields an infinite sequence of triples $\left(X_{i}, Y_{i}, Z_{i}\right)(i=1,2, \ldots)$ such that each finite initial subsequence satisfies the conditions of the lemma. Note that the $X_{i}$ s all convert to different numerals. Define (classically!) $\left(n, Y_{n}^{\prime}, Z_{n}^{\prime}\right)$ to be $\left(X_{i}, Y_{i}, Z_{i}\right)$ if $X_{i}=\underline{n}$ for some $i$, and $\left(\underline{n}, \underline{0}_{\tau}\right.$, Axiom $\left._{1} \underline{n}\right)$ otherwise. This results in an infinite sequence $\not_{n} n$. $\left(\underline{n}, Y_{n}^{\prime}, Z_{n}^{\prime}\right)$ such that $\not{\not} n$. $Y_{n}^{\prime}$ is reducible and $\chi_{n} n . Z_{n}^{\prime}$ realizes $\forall x: \mathrm{N} \neg \phi\left(x,\left(\not{M n} n . Y_{n}^{\prime}\right) x\right)$. It follows that $P\left(\not{\not} n . Y_{n}^{\prime}, \not{M} n . Z_{n}^{\prime}\right)$ realizes $\perp$ and hence reduces to a term of the form Axiom ${ }_{i} \underline{k}$. By Fact $(\mathrm{v})$ from 3.4, the Continuity Lemma, $P\left(\not{Z n} n . Y_{n}^{\prime}, \not{X} n . Z_{n}^{\prime}\right)$ only depends on a finite initial subsequence of $\not{X} n .\left(\underline{n}, Y_{n}^{\prime}, Z_{n}^{\prime}\right)$. This conflicts with the above construction of $\left[\left(X_{1}, Y_{1}, Z_{1}\right), \ldots,\left(X_{n}, Y_{n}, Z_{n}\right)\right]$ for $n$ large enough.

4.6. Comments on this interpretation. This interpretation gives a quite direct computational interpretation of the Axiom of Choice. For instance, it allows to interpret directly the first informal proof of Higman's lemma presented in [17], and avoids in this case the encoding of a function as a relation. Observe furthermore that the computation is demand-driven in the sense that the list of triples only contains function values and realizers that are really needed for the computation of a realizer of $\perp$.

It should be noted also that our interpretation works for $\operatorname{AC}\left(\tau, \tau^{\prime}\right)$ where $\tau=N$ but $\tau^{\prime}$ is arbitrary. As it is given, it uses in an essential way the restriction $\tau=\mathbf{N}$, but we shall present below heuristic arguments that suggest a possible extension to higher types.

Already in the case $A C(N, N \rightarrow N)$, the encoding of a function as a relation used in [17] to avoid the use of $A C(N, N)$ does not work any more. The fact that the Axiom of Choice $A C(N, N)$ is validated by considering functions as relations indeed uses the existence of a well founded total ordering on $N$, but we don't know of any such effective ordering on $\mathrm{N} \rightarrow \mathrm{N}$. 
§5. Axiom of Dependent Choice. We realize now the negative translation of $\mathrm{DC}_{\tau}$, the axiom of Dependent Choice at type $\tau$ :

$[\forall n: \mathrm{N} \forall x: \tau \exists y: \tau \phi(n, x, y)] \Longrightarrow \exists f: \mathrm{N} \rightarrow \tau \forall n: \mathrm{N} \phi(n, f(n), f(n+1))$,

which, as explained in [11], implies directly the usual versions of the Axiom of Choice and of the axiom of Dependent Choice. Below it is implicit that $x$ and $y$ are of the same type $\tau, n$ of type $N$ and that the function $f: \mathbf{N} \rightarrow \tau$ is such that $f(0)=a$, where $a$ is some fixed object of type $\tau$.

The axiom of Dependent Choice is commonly used in informal mathematics. As proved in [11], $A C(N, N)$ trivially implies $D C_{N}$ by primitive recursion. For higher types $\tau$, the axiom of Dependent Choice appears to be stronger than the Axiom of Choice. (In [11], Problem 9 asks whether $A C(N, N \rightarrow N)$ implies $D C_{N \rightarrow N}$, and we do not know if this question is still open.)

The negative translation of this schema is subsumed by the following axiom schema K DC:

$$
[\forall n \forall x \nabla \exists y \neg \phi(n, x, y)] \Longrightarrow \nabla \exists f \forall n \neg \phi(n, f(n), f(n+1)) .
$$

We shall give a program that realizes this schema. Furthermore, we prove this fact not using classical logic and dependent choices, but intuitionistic meta-reasoning together with a certain strong principle of bar induction.

This interpretation is based on the same idea of partial approximation of a function. An approximation of $f$ satisfying $\forall n \neg \phi(n, f(n), f(n+1))$ will be given as a term $u=\left(n, f_{n}, l\right)$ where $n$ is a numeral, $f_{n}$ a term of type $\tau$ and $l$ a list of the form

$$
\left[\left(0, a, q_{0}\right), \ldots,\left(n-1, f_{n-1}, q_{n-1}\right)\right]
$$

such that $q_{i}$ is of type $\left|\neg \phi\left(i, f_{i}, f_{i+1}\right)\right|$ for $i<n$ (with the convention that $f_{0}=a$, the fixed object of typed $\tau$ ). It is now standard to redefine fun and get such that

fun $u i$

is $f_{i}$ if $i \leq n$, and $0_{\tau}$ if $i>n$, and that

get $u$ i $b$

is $q_{i}$ if $i<n$, and $b$ if $n \leq i$.

If $u=\left(n, f_{n}, l\right)$ is such an approximation, and $f$ is of type $\tau$ and $q$ of type $\left|\neg \phi\left(n, f_{n}, f\right)\right|$, we write $u+(f, q)$ for the approximation $\left(n+1, f,\left(n, f_{n}, q\right): l\right)$. We say that $v$ is a direct extension of $u$ if $v$ is of the form $u+(f, q)$ for some $f, q$, and we denote this by $u<v$.

We define recursively, when $u$ is of the form $\left(n, f_{n}, l\right)$

$\Phi P H u=P\left(\right.$ fun $u$, $\lambda i$.get $u i\left(\lambda x^{\prime}:|\phi| . H(n+1)(\lambda(y, z)\right.$. $\left.\left.P H(u+(y, z)))\right)\right)$.

It is then possible to show that $\lambda h \lambda p . \Phi p h(0, a,[])$ realizes K DC, by a classical argument similar to the one we gave for the negative translation of the Axiom of Choice.

For an intuitionistic reduction to the principle of bar induction, we introduce

$$
\Phi^{\prime} P H u=P\left(\text { fun } u, \lambda i \text {.get } u i\left(\lambda x^{\prime}:|\phi| \text {.Axiom } 1 i\right)\right) \text {. }
$$

As before, we can suppose that Axiom 1 does not occur in $P$ nor in $H$. 
We define a reducible approximation as an approximation $\left(n, f_{n}, l\right)$ such that $f_{n}$ is a reducible term of type $\tau$ and $l$ is a list

$$
\left[\left(0, a, q_{0}\right), \ldots,\left(n-1, f_{n-1}, q_{n-1}\right)\right]
$$

such that $f_{i}$ is a reducible term of type $\tau$ and $q_{i}$ realizes $\neg \phi\left(i, f_{i}, f_{i+1}\right)$ for $i<n$, with $f_{0}=a$. Furthermore we ask that Axiom 1 does not appear neither in $f_{n}$ nor in $l$.

Note that, for each reducible approximation $u$, the term $\Phi^{\prime} P H u$ realizes $\perp$. Furthermore, by a reasoning similar to the one of the previous section, if $\Phi^{\prime} P H u$ does not reduce to a term of the form Axiom $n_{0}$, then we have $\Phi P H u=\Phi^{\prime} P H u$ and so $\Phi P H u$ realizes $\perp$. A natural terminology is to say that the approximation $u$ is partial if $\Phi^{\prime} P H u$ reduces to a term of the form Axiom $n_{0}$, and complete otherwise.

We claim that the set of complete approximations is a bar on the set of reducible approximations. This follows from the fact that any sequence of reducible approximations $u_{0}<u_{1}<u_{2}<\ldots$ is eventually complete. That is, there exists $n_{0}$ such that $u_{n}$ is complete if $n_{0} \leq n$. This is established using the Continuity Lemma as in the previous section.

It is now direct to prove by bar induction that $\Phi P H u$ realizes $\perp$ if $u$ is a reducible approximation. If $u<v$ implies that $\Phi P H v$ realizes $\perp$, it follows indeed from the definition of $\Phi$ that $\Phi P H u$ realizes $\perp$.

§6. Comparison with Spector's work. In [6] Gödel introduced the so-called Dialectica interpretation, a translation of intuitionistic arithmetic into $H A^{\omega}$, which satisfies the following preservation property. If $\phi$ is a theorem of intuitionistic arithmetic, then $\phi^{D} \equiv \exists f \forall x \phi_{D}$, with $\phi_{D}$ quantifier-free, can be validated in $\mathrm{HA}^{\omega}(f$ and $x$ may be sequences of variables). This means that there exists a closed term $F$ such that $\forall x \phi_{D}[f:=F]$ is a theorem of $\mathrm{HA}^{\omega}$. The types of $f$ and $x$ only depend on the logical structure of $\phi$, and $F$ depends on the proof of $\phi$. Thus the Dialectica interpretation absorbes the quantifiers of $\phi$ at the cost of higher types in $\exists f \forall x \phi_{D}$.

In [22] Spector extended the Dialectica interpretation to analysis, by interpreting Double Negation Shift, DNS for short. DNS is the following axiom schema

$$
[\forall x: N \nabla \phi(x)] \Longrightarrow \nabla \forall x: \mathrm{N} \phi(x) \text {. }
$$

It is direct to show that this schema plus the Axiom of Choice implies intuitionistically the negative translation of the Axiom of Choice. An alternative approach is taken by Howard in [10], who first shows that the theory $H^{\omega}$ extended with the axiom schema $\phi \Longleftrightarrow \phi^{D}$ plus bar induction has a bar recursive Dialectica interpretation, and then shows that $K A C$ and $K D C$ are theorems of this extended theory.

The crucial step, where [22] goes beyond [6], is the Dialectica interpretation of DNS, which leads to the following formula.

$$
\forall a y d \exists n z f[\phi(n, a n z, z(a n z)) \Longrightarrow \phi(y f, f(y f), d f))]
$$

Here the type of $n$ is $\mathrm{N}$, the type of $f$ is $\mathrm{N} \rightarrow \tau$ and the types of the other variables can easily be inferred. The problem is to find closed terms yielding $n, z, f$ when applied to $a, y, d$. This will be done by bar recursion. 
Spector solved the system of functional equations arising from (3), i.e.,

$$
\forall a y d \exists n z f[n=y f \wedge a n z=f(y f) \wedge z(a n z)=d f]
$$

by first reducing it to

$$
\forall a y d \exists f \forall n \leq y f \exists z_{n}\left[a n z_{n}=f n \wedge z_{n} f n=d f\right]
$$

and then solving (5) by bar recursion. Clearly (5) implies (4) by taking $n=y f$ and $z=z_{n}$.

We introduce the following abbreviation

$$
\phi(n, f) \equiv n \leq y f \Longrightarrow \exists z_{n}\left[a n z_{n}=f n \wedge z_{n} f n=d f\right] .
$$

Let $A, Y, D$ be given. We shall define by bar recursion a functional $\Phi$ which extends every list $L=\left[F_{m-1}, \ldots, F_{0}\right]$ to a functional $F=\Phi L$ satisfying $F i=F_{i}$ for all $i<m$ and $\forall n \geq m \phi(n, F)$. Then $F=\Phi[]$ solves (5). Assume such $\Phi(X: L)$ has already been defined for all $X$. We are looking for a definition of $\Phi L$. We redefine fun $L$ as the function which extends $L$ with infinitely many function values $\underline{0}_{\tau}$. If $Y$ (fun $\left.L\right)<m$, then we put $\Phi L=$ fun $L$ and $F=\Phi L$ vacuously satisfies $\forall n \geq m \phi(n, F)$, since $n>Y F$ for all $n \geq m$. Now suppose $Y($ fun $L) \geq m$. By assumption we have for any $X$ that $F=\Phi(X: L)$ satisfies $\forall n \geq m+1 \phi(n, F)$. So if we put $\Phi L=\Phi\left(F_{m}: L\right)$, then it only remains to determine a suitable $F_{m}$ such that $F=\Phi\left(F_{m}: L\right)$ satisfies $\phi(m, F)$, i.e., $\exists z_{m}\left[A m z_{m}=F m \wedge z_{m} F m=D F\right]$. Take to this end $F_{m}=A m Z_{m}$ with $Z_{m}=\lambda x . D(\Phi(x: L))$, then indeed $F m=F_{m}=A m Z_{m}$ and $Z_{m}(F m)=Z_{m}\left(F_{m}\right)=D\left(\Phi\left(F_{m}: L\right)\right)=D F$. This completes the definition of $\Phi$, and hence (5) is solved.

If we resume the definition of $\Phi$, then we get:

$$
\Phi \text { a } y d l= \begin{cases}\text { fun } l & \text { if } y(\text { fun } l)<m \\ \Phi a y d(\operatorname{am}(\lambda x . d(\Phi a y d(x: l))): l) & \text { otherwise }\end{cases}
$$

with $m$ the length of the list $l$, which is an instance of the definition schema of bar recursion (see [22], [10]). Using $\Phi$ it is easy to find closed terms yielding $n, z, f$ when applied to $a, y, d$, or in other words to solve (3).

There is an important difference in motivation between the work of Spector and ours. Spector's aim was to prove the consistency of analysis and to characterize its proof theoretic strength in terms of a subrecursive calculus. To this end, bar recursion suits well. Our aim is to explore the computational content of various choice axioms in combination with classical logic in order to extract algorithms from proofs. To this end, the Dialectica interpretation is rather indirect, and the realizability interpretation suits better.

§7. Game semantics. The purpose of this informal section is to explain how the program was found that realizes the negative translation of the Axiom of Choice. The importance of this section is that it may indicate a connection with the work on sequential algorithms, and may pave the way for a generalization to the case of the Axiom of Choice at higher-types, i.e., the axiom $\operatorname{AC}\left(\tau, \tau^{\prime}\right)$ with $\tau \neq N$. 
7.1. A semantics of classical arithmetic. The first step is to give an intuitive interpretation of cut-free provability as defined by Novikoff [18]. This is defined for an infinitary propositional calculus. Each arithmetical formula can be naturally represented in such a calculus. For instance $\forall k \exists n \geq k \forall m \geq k f(n) \leq f(m)$, which expresses that any function $f$ has a minimum among its values $f(n)$ for $k \leq n$, becomes the propositional formula

$$
\bigwedge_{k} \bigvee_{n \geq k} \bigwedge_{m \geq k} \phi_{n m}
$$

where $\phi_{n m}$ is, for given numerals $n$ and $m$, the truth value of the (decidable) formula $f(n) \leq f(m)$.

The formulae are defined inductively by

- 0 and 1 are formulae,

- if $\phi_{i}$ is a family of formulae over a countable decidable set, then $\bigwedge_{i} \phi_{i}$ and $\bigvee_{i} \phi_{i}$ are formulae.

Iterated conjunctions (disjunctions) are packed together as one single conjunction (disjunction), so that in every formula conjunctions and disjunctions are alternating. The notion of intuitionistic validity

- 1 is valid,

- $\bigvee_{i} \phi_{i}$ is valid if and only if there exists $i_{0}$ such that $\phi_{i_{0}}$ is valid,

- $\bigwedge_{i} \phi_{i}$ is valid if and only if $\phi_{i}$ is valid for all $i$,

has a natural game theoretical interpretation. The game is played by two players, $\forall$ belard and $\exists$ loise, who quarrel about the truth of a given formula. The player $\exists$ loise, who argues in favour, plays when the formula is existential, say $\bigvee_{i} \phi_{i}$, by choosing an index $i_{0}$ ("ᄏloise's Choice"), after which the game is continued with the formula $\phi_{i_{0}}$. The player $\forall$ belard, who argues against, plays when the formula is universal, say $\bigwedge_{j} \phi_{j}$, by choosing an index $j_{0}$, after which the game is continued with the formula $\phi_{j_{0}}$. The game ends when either $\forall$ belard or $\exists$ loise chooses the formula 0 or 1 . Evidently, the game always terminates. If either $\exists$ loise or $\forall$ belard chooses 1 , then $\exists$ loise wins the game and $\forall$ belard loses. Dually, if either $\exists$ loise or $\forall$ belard chooses 0 , then $\forall$ belard wins the game and $\exists$ loise loses.

We have the following completeness result: the player $\exists$ loise has a winning strategy for the game associated to the existential formula $\phi$ if and only if this formula $\phi$ is intuitionistically valid. (Every formula is equivalent to an existential formula by prefixing it with a singleton disjunction.)

For instance, there is no winning strategy for the formula

$$
\bigwedge_{k} \bigvee_{n \geq k} \bigwedge_{m \geq k} f(n) \leq f(m)
$$

because it is not possible to compute effectively such a minimum value for a function $f$.

The notion of classical validity, as described by Novikoff [18], is inductively defined by the clauses

- 1 is valid,

- $\bigvee_{i} \phi_{i}$ is valid if and only if there exists $i_{0}$ such that $\phi_{i_{0}}$ is 1 , or is an universal formula $\bigwedge_{j} \phi_{i_{0} j}$ such that, for all $j$, the formula $\phi_{i_{0} j} \vee \bigvee_{i} \phi_{i}$ is valid, 
- $\bigwedge_{i} \phi_{i}$ is valid if and only if $\phi_{i}$ is valid for all $i$.

This definition may seem not well founded, but it correctly defines classical validity as the smallest set satisfying the above closure properties.

For instance, a formula of the form $\psi \vee \bigvee_{i} \phi_{i} \vee \overline{\phi_{i_{0}}}$ is always valid. This is shown by induction on the formula $\bigvee_{i} \phi_{i}$. First, recall that $\bar{\phi}$ is the usual defined complement of $\phi$, changing $\bigvee s$ into $\Lambda \mathrm{s}, 1 \mathrm{~s}$ in $0 \mathrm{~s}$, and conversely. Second, $\phi_{i_{0}}$ is either 0 or 1 , or a universal formula $\bigwedge_{j} \phi_{i_{0} j}$ by the alternation of $\bigvee \mathrm{s}$ and $\Lambda \mathrm{s}$. In the first two cases, it is trivial to choose the index of a valid disjunct in the formula above. In the third case, choose the index such that we get the formula $\psi \vee \bigvee_{i} \phi_{i} \vee \overline{\phi_{i_{0}}} \vee \phi_{i_{0} j}$, which is valid for all $j$ by the induction hypothesis.

The game semantics has to be adapted to the classical notion of validity: in the case of a disjunction $\bigvee_{i} \phi_{i}$, the game proceeds with the formula $\bigwedge_{j}\left[\phi_{i_{0} j} \vee \bigvee_{i} \phi_{i}\right]$. The other cases do not change. A tentative interpretation of this definition is that it describes exactly a winning strategy for a game between $\forall$ belard and $\exists$ loise. This strategy is suggested by the inductive definition of classical validity: in the case of an existential formula, and after a choice $j_{0}$ of $\forall$ belard, ᄏloise should try again and choose a new $i$ in

$$
\phi_{i_{0} j_{0}} \vee \bigvee_{i} \phi_{i}
$$

using information drawn from $\phi_{i_{0} j_{0}}$ (if this latter formula is 1 , then $\exists$ loise should choose this formula, of course).

There is such a winning strategy for the formula

$$
\bigwedge_{k} \bigvee_{n \geq k} \bigwedge_{m \geq k} \phi_{n m}
$$

with $\phi_{n m}=f(n) \leq f(m)$, which can be described as follows. The game starts by $\forall$ belard giving a value $k$. The player $\exists$ loise answers choosing an arbitrary value $n \geq k$, say $n=k$ and $\forall$ belard answers by playing $m=m_{0} \geq k$. The formula becomes

$$
\phi_{k m_{0}} \vee \bigvee_{n \geq k} \bigwedge_{m \geq k} \phi_{n m}
$$

If $\phi_{k m_{0}}$ is true, then $\exists$ loise wins. If $\phi_{k m_{0}}$ is false, the strategy for $\exists$ loise is to choose the last value given by $\forall$ belard. After $\forall$ belard chooses a value $m=m_{1} \geq k$, the formula becomes

$$
\phi_{k m_{0}} \vee \phi_{m_{0} m_{1}} \vee \bigvee_{n \geq k} \bigwedge_{m \geq k} \phi_{n m}
$$

The player $\exists$ loise wins if $f\left(m_{0}\right) \leq f\left(m_{1}\right)$. In this way, to each game corresponds a sequence $m_{0}, m_{1}, \ldots$ such that $\phi_{k m_{0}}, \phi_{m_{0} m_{1}}, \ldots$ are all false, i.e., such that $f\left(m_{0}\right)>f\left(m_{1}\right)>\ldots$ Since $\mathrm{N}$ is well founded, Jloise will win eventually by following this strategy.

7.2. Game semantics and realizability. It is possible to represent $\exists$ loise's strategy by a program that realizes the negative translation of the formula $\forall k \exists n \geq k \forall m \geq k$ $f(n) \leq f(m)$, which is $\forall k \nabla \exists n \geq k \forall m \geq k \nabla f(n) \leq f(m)$. We make the 
(inessential) simplification that $k=0$. Consider the following program:

$$
\Phi P n=P\left(n, \lambda m \lambda h \text {. if } \phi_{n m} \text { then }(h()) \text { else }(\Phi P m)\right) \text {, }
$$

where () is the only constant of type Unit. It is possible to show that $\lambda p . \Phi p 0$ realizes the formula $\nabla \exists n \forall m \nabla f(n) \leq f(m)$. This program follows closely the previous strategy of $\exists$ loise, and the proof that $\lambda p . \Phi p 0$ realizes the formula $\nabla \exists n \forall m \nabla f(n) \leq f(m)$ is similar to the argument showing that this strategy is winning.

7.3. A strategy for the Axiom of Choice. In [5] it was conjectured that it should be possible to extend this interpretation in the case of quantification over functions. The idea would be simply to allow as index set the set of all functions, and, apart from this single change, to keep the same notion of games and strategies. This suggested the realization of the Axiom of Choice given above.

There is an essential difficulty, closely connected to the problem in sequent calculus mentioned above, that we cannot require the strategy for $\exists$ loise to win eventually against any player $\forall$ belard. For instance, let us consider the classically valid formula

$$
\bigvee_{g} \bigwedge_{k} \bigwedge_{m \geq k} f(g(k)) \leq f(m),
$$

where $g$ should be a function such that $k \leq g(k)$ for all $k$. It should be clear that $\forall$ belard may force the game to be infinite by playing successively $k=0,1,2, \ldots$ since at each move $\exists$ loise can only hope to update the value $g(k)$.

To analyse this difficulty, we shall consider the more general case of a gametheoretic justification of the Axiom of Choice

$$
\mathrm{AC}=\bigvee_{m} \bigwedge_{x} \bigwedge_{y} \overline{\phi_{m x y}} \vee \bigvee_{f} \bigwedge_{m} \bigvee_{y} \phi_{m f(m) y}
$$

In the sequel, $\phi_{m x}$ abbreviates $\bigvee_{y} \phi_{m x y}$; we have taken the instance of AC with this latter formula in order to have alternating quantifiers in case $\phi_{m x}$ is universal. The player $\exists$ loise has the following natural strategy. $\exists$ loise starts by playing an arbitrary function, for instance the constant function $f_{0}=\lambda x .0$. The player $\forall$ belard answers by a value $m=m_{0}$ and the formula becomes

$$
\phi_{m_{0} 0} \vee A C .
$$

The player $\exists$ loise can then play $m=m_{0}$, and $\forall$ belard answers with two values $x=x_{0}$ and $y=y_{0}$. The formula becomes

$$
\overline{\phi_{m_{0} x_{0} y_{0}}} \vee \phi_{m_{0} 0} \vee A C \text {. }
$$

The player $\exists$ loise backtracks by choosing $f_{1}=f_{0}+\left[m_{0} \longmapsto x_{0}\right]$ which is defined by $f_{1}(m)=f_{0}(m)$ for $m \neq m_{0}$ and $f_{1}(m)=x_{0}$ for $m=m_{0}$. We can suppose that $\forall$ belard will answer with a value $m_{1} \neq m_{0}$. Indeed, if $m_{1}=m_{0}$, then the formula will have the form

$$
\overline{\phi_{m_{0} x_{0} y_{0}}} \vee \phi_{m_{0} x_{0}} \vee \ldots
$$

and we have seen above that $\exists$ loise has always a winning strategy for this kind of formulae. 
In this way, we can associate to this game a successive updating of the function $f_{0}$

$$
f_{k}=f_{0}+\left[m_{0} \longmapsto x_{0}\right]+\left[m_{1} \longmapsto x_{1}\right]+\cdots+\left[m_{k} \longmapsto x_{k}\right]
$$

where $m_{j+1}$ is $\forall$ belard's answer to the move $f=f_{j}$ of $\exists$ loise.

The crucial point now is that $\forall$ belard loses as soon as we have $m_{k+1}=m_{j}$ for some $j \leq k$.

If we assume that the player $\forall$ belard can only base his move on finitely many information from the move $f=f_{k}$ of $\exists$ loise, this will happen eventually, by a continuity argument.

Hence, if we change our notion of winning strategy in asking only that the player $\exists$ loise has to win eventually against any "finite" player $\forall$ belard, this strategy is a winning strategy for the formula $A C$. The program that realizes the negative translation of the Axiom of Choice is a direct translation of this strategy. As in the arithmetical case, the proof of reducibility of this program closely follows the argument that such a strategy is "winning" against any "reasonable" player $\forall$ belard.

This argument is only yet heuristic and the main problem is making precise what is a "finite" player $\forall$ belard. We have seen that it would be wrong to ask that it wins against any, non-computable, strategy. But it is probably too restrictive to ask that it wins eventually against any computable strategy. One may try to define a winning strategy for $\exists$ loise as a strategy winning eventually against any continuous strategy for $\forall$ belard. We avoided here this difficulty by formulating the interpretation in a functional system, but we can hope for a much more perspicuous analysis of the computation if it is expressed as an interaction between strategies.

Another possibility would be to use sequential algorithms for representing functions. To give a function will then not be an atomic operation, but the function will in general be given "piece by piece". One may then hope to avoid the consideration of continuity.

§8. Conclusion. There are many directions in which this work can be improved and some potential connections that seem worthwhile to analyse.

One is the formulation of this work in the framework of sequential algorithms, which is indeed the natural framework in which one can observe "intensional behaviour" of functionals, which has been our main tool in the realization of the negative translation of the Axiom of Choice. We expect from such a formulation a much more transparent proof of termination (and it will be interesting to see what becomes in this formalism the difficulty of finding "fresh" constants). We also think that in such a framework, the generalization to higher types, that is, a computational interpretation of $\mathrm{AC}\left(\tau, \tau^{\prime}\right)$ for arbitrary $\tau$ and $\tau^{\prime}$, should be straightforward. An elegant form would be an interpretation of the notion of dependent types in the framework of sequential algorithms, together with a direct interpretation of $\nabla \phi$, and the inference of $\nabla \forall x: \tau . \phi(x)$ from $\forall x: \tau . \nabla \phi(x)$.

Closely related should be the question of a constructive formulation of our proof of realizability. Can we adapt the method of [2] and avoid the use of infinite terms? Our hope is that our interpretation, computationally more direct than the one of 
Spector $[22,10]$, may provide help for a constructive understanding of classical analysis with the Axiom of Choice.

Experiments with some examples, in particular Higman's Lemma, have revealed a computational inefficiency of our interpretation. Intuitively, our algorithms proceed by trial and errors and may "forget too many things" of previous trials. It seems possible to design improved algorithms, that "remember" and can use all previous trials. This problem is closely connected to the problem of the computational interpretation of the implication $\nabla \phi \Longrightarrow \nabla \psi \Longrightarrow \nabla(\phi \wedge \psi)$ so that its solution should have consequences even for the problem of the computational content of propositional classical logic.

The previous remark, and metaphors such as "trials and errors", "conjecturing laws that are refined by experimentation", are immediately suggested by trying to understand the computational behaviour of our interpretation. They hint at possible connections with learning theories, such as the one described in [19], where the learning agent may benefit from negative information.

Yet another natural connection is with the work of Hilbert $[8,9]$ and Ackermann [1]. Our interpretation can be seen as a variation of Hilbert's epsilon method $[8,9]$, with a (classical) proof of termination. It will be interesting to compare our algorithm with the one described in Ackermann's paper [1]. ${ }^{4}$

Acknowledgement. We thank Anne Troelstra for helpful comments and answers to various questions that arose during the preparation of this paper, Loïc Colson for interesting discussions on the axiom of choice and impredicativity at the beginning of this work, and Marco Hollenberg, Erik Barendsen and Jan Springintveld for carefully reading successive draft versions.

\section{REFERENCES}

[1] W. ACKeRMANN, Begründung des Tertium non datur mittels der Hilbertschen Theorie der Widerspruchsfreiheit, Mathematische Annalen, vol. 93 (1924), pp. 1-36.

[2] M. BEZEM, Strong normalization of barrecursive terms without using infinite terms, Archiv für mathematische Logik und Grundlagenforschung, vol. 25 (1985), pp. 175-182.

[3] E. BISHOP, Foundations of constructive analysis, McGraw-Hill, New York, 1967.

[4] R. CONSTABLE and C. MURTHY, Finding computational content in classical proofs, Logical frameworks (G. Huet and G. Plotkin, editors), Cambridge University Press, 1991, pp. 341-362.

[5] Th. Coquand, A semantics of evidence for classical arithmetic, this Journal, vol. 60 (1995), pp. $325-337$.

[6] K. GöDel, Collected work, vol. I and II (S. Feferman, J. W. Dawson, S. C. Kleene, G. H. Moore, R. M. Solovay, and J. van Heijenoort, editors), Oxford, 1986.

[7] N. Goodman, Intuitionistic arithmetic as a theory of constructions, Ph.D. thesis, Stanford University, 1968.

[8] D. Hilbert, Die logischen Grundlagen der Mathematik, Mathematische Annalen, vol. 88 (1923), pp. $151-165$.

[9] D. HilberT, The foundations of mathematics, From Frege to Gödel (J. van Heijenoort, editor), Harvard University Press, Cambridge, MA, 1971, pp. 465-479.

[10] W. A. HowARD, Functional interpretation of bar induction by bar recursion, Compositio Mathematica, vol. 20 (1968), pp. 107-124.

\footnotetext{
${ }^{4}$ According to [15], if it is well-known that the proof of convergence of Ackermann's algorithm was defective as presented in [1], it is not known yet, not even non-constructively, if the method converges at all.
} 
[11] W. A. HowARD and G. KREISEL, Transfinite induction and bar induction of types zero and one, and the role of continuity in intuitionistic analysis, this JouRnAL, vol. 31 (1966), pp. 325-358.

[12] S. C. KLEENE, On the interpretation of intuitionistic number theory, this JouRNAL, vol. 10 (1945), pp. 109-124.

[13] A. N. Kolmogorov, On the principle of the excluded middle, From Frege to Gödel (J. van Heijenoort, editor), Harvard University Press, Cambridge, MA, 1971, pp. 465-479.

[14] G. KREISEL, On weak completeness of intuitionistic predicate logic, this JoURNaL, vol. 27 (1962), pp. $139-158$

[15] - Mathematical logic, Lectures on modern mathematics (Saaty, editor), vol. III, Wiley, 1965, pp. 95-195. 1982.

[16] G. E. MOORE, Zermelo's axiom of choice: Its origins, development and influence, Springer-Verlag,

[17] C. MurThy, Extracting constructive content from classical proofs, Ph.D. thesis, Cornell University, 1990.

[18] P. S. Novikoff, On the consistency of certain logical calculi, Matematiceskij sbornik (Recueil Mathématique), vol. 12 (1943), no. 54, pp. 230-260.

[19] D. N. Osherbon, M. Stob, and S. WeInStein, Systems that learn: An introduction to learning theory for cognitive and computer scientists, MIT Press, 1986.

[20] D. SCOTT and A. TARSKI, The sentential calculus with infinitely long expressions, Colloquium Mathematicum, vol. VI (1958), pp. 165-170.

[21] J. R. ShOENFIELD, Mathematical logic, Addison-Wesley, 1967.

[22] C. SPECTOR, Provably recursive functionals of analysis: A consistency proof of analysis by an extension of principles formulated in current intuitionistic mathematics, Recursive function theory (J. C. E. Dekker, editor), Proceedings of Symposia in Pure Mathematics, no. V, American Mathematical Society, 1961, pp. 1-27.

[23] W. W. TAIT, Normal derivability in classical logic, Lecture notes in mathematics (J. Barwise, editor), no. 72, Springer-Verlag, Berlin, 1968, pp. 204-236.

[24] - Normal form theorem for bar recursive functions of finite type, Proceedings of the second scandinavian logic symposium (J. E. Fenstad, editor), North-Holland, Amsterdam, 1971, pp. 353-367.

[25] A. S. Troelstra, Realizability, ILLC Prepublication Series for Mathematical Logic and Foundations ML-92-09.

[26] - Metamathematical investigation of intuitionistic arithmetic and analysis, Lecture Notes in Mathematics, no. 344, Springer-Verlag, Berlin, 1973.

[27] - A note an non-extensional operations in connection with continuity and recursiveness, Indagationes Mathematicae, vol. 39 (1977), pp. 455-462.

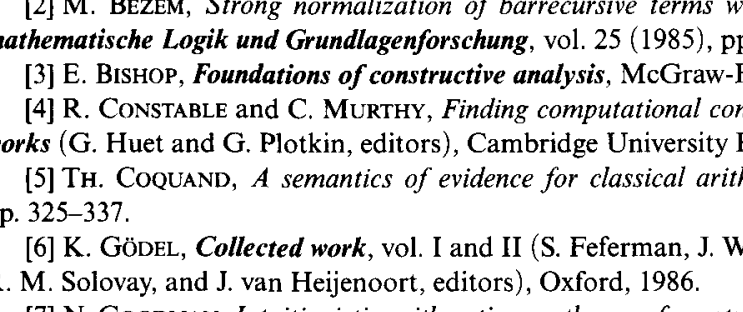

E-mail: coquand@cs.chalmers.se 
[11] W. A. HowARD and G. KREISEL, Transfinite induction and bar induction of types zero and one, and the role of continuity in intuitionistic analysis, this JouRnAL, vol. 31 (1966), pp. 325-358.

[12] S. C. KLEENE, On the interpretation of intuitionistic number theory, this JouRNAL, vol. 10 (1945), pp. 109-124.

[13] A. N. Kolmogorov, On the principle of the excluded middle, From Frege to Gödel (J. van Heijenoort, editor), Harvard University Press, Cambridge, MA, 1971, pp. 465-479.

[14] G. KREISEL, On weak completeness of intuitionistic predicate logic, this JoURNaL, vol. 27 (1962), pp. $139-158$

[15] - Mathematical logic, Lectures on modern mathematics (Saaty, editor), vol. III, Wiley, 1965, pp. 95-195. 1982.

[16] G. E. MOORE, Zermelo's axiom of choice: Its origins, development and influence, Springer-Verlag,

[17] C. MurThy, Extracting constructive content from classical proofs, Ph.D. thesis, Cornell University, 1990.

[18] P. S. Novikoff, On the consistency of certain logical calculi, Matematiceskij sbornik (Recueil Mathématique), vol. 12 (1943), no. 54, pp. 230-260.

[19] D. N. Osherbon, M. Stob, and S. WeInStein, Systems that learn: An introduction to learning theory for cognitive and computer scientists, MIT Press, 1986.

[20] D. SCOTT and A. TARSKI, The sentential calculus with infinitely long expressions, Colloquium Mathematicum, vol. VI (1958), pp. 165-170.

[21] J. R. ShOENFIELD, Mathematical logic, Addison-Wesley, 1967.

[22] C. SPECTOR, Provably recursive functionals of analysis: A consistency proof of analysis by an extension of principles formulated in current intuitionistic mathematics, Recursive function theory (J. C. E. Dekker, editor), Proceedings of Symposia in Pure Mathematics, no. V, American Mathematical Society, 1961, pp. 1-27.

[23] W. W. TAIT, Normal derivability in classical logic, Lecture notes in mathematics (J. Barwise, editor), no. 72, Springer-Verlag, Berlin, 1968, pp. 204-236.

[24] - Normal form theorem for bar recursive functions of finite type, Proceedings of the second scandinavian logic symposium (J. E. Fenstad, editor), North-Holland, Amsterdam, 1971, pp. 353-367.

[25] A. S. Troelstra, Realizability, ILLC Prepublication Series for Mathematical Logic and Foundations ML-92-09.

[26] - Metamathematical investigation of intuitionistic arithmetic and analysis, Lecture Notes in Mathematics, no. 344, Springer-Verlag, Berlin, 1973.

[27] - A note an non-extensional operations in connection with continuity and recursiveness, Indagationes Mathematicae, vol. 39 (1977), pp. 455-462.

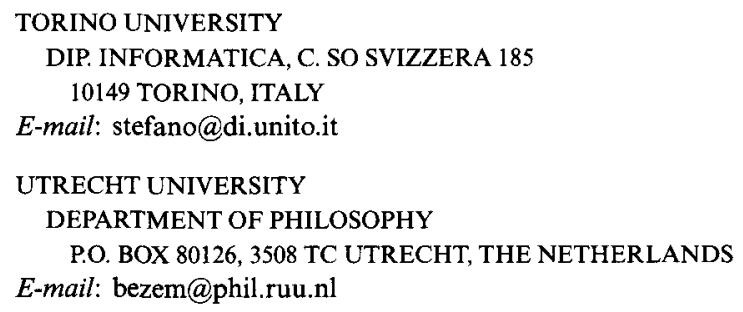

E-mail: coquand@cs.chalmers.se 